\title{
Effect of Kangaroo Mother Care on Heart rate, Respiratory rate and Temperature in Low Birth Weight Babies
}

\author{
Verma $\mathbf{P}^{1}$, Verma $\mathbf{V}^{2}$ \\ ${ }^{1}$ Dr Pramila Verma, Assistant professor, Department of paediatrics, Peoples college of Medical Sciences, Bhopal, India, ${ }^{2} \mathrm{Dr}$ \\ Vijay Verma, Department of Radiodiagnosis, Gandhi Medical College, Bhopal, India
}

Address for correspondence: Dr. Pramila Verma, Email: drpramilav@yahoo.com

\begin{abstract}
Background: Kangaroo mother care (KMC) involves skin-to-skin positioning of the infant in an upright position against the mother's chest. The two other components of KMC are frequent and exclusive breastfeeding (where possible) and early discharge from hospital. Aim of present study was to asses effect of kangaroo mother care on heart rate, respiration rate and temperature of low-birth weight preterm newborns admitted in a tertiary care centre of central India. Methods: Low birth weight babies (weighing < 2000 grams) were given kangaroo mother care (KMC) for 30minutes. Pre and post KMC heart rate, respiratory rate and temperature were measured. The evaluations were done once a day, for three consecutive days and the mean values were taken for analysis. The data was statistically analyzed using plus 6.0 statistical software. Student $t$ test was used to find out the statistical difference between two mean SD. Results: Results of the study reveal that changes in heart rate and respiration rate after application of kangaroo care was insignificant $(\mathrm{p}>0.05)$ where as kangaroo care is highly effective in maintaining skin temperature $(\mathrm{p}<0.0005)$. Conclusion: $\mathrm{KMC}$ maintains heart rate, respiratory rate and temperature in a stable preterm low birth weight babies.
\end{abstract}

Key words: Kangaroo care, KMC, Heart rate, LBW

\section{Introduction}

Low birth weight for our country is a serious public health problem and contributes to high neonatal mortality. According to the report published in Lancet almost all (99\%) neonatal deaths occur in low income and middleincome countries [1, 2]. In India around one million babies die each year before they complete their first month of life, contributing to one-fourth of the global burden [1, 3]. Prematurity is one of the common causes of neonatal deaths in developing countries like India. These neonates need special care as they have several handicaps, the most important being maintenance of body temperature. To overcome this problem kangaroo care was introduced in Bogota, Colombia, more than 25 years ago as one component of an alternative approach to traditional neonatal intensive care unit (NICU) care for low- birth weight infants [4]. Kangaroo mother care (KMC) is the practice of skin-toskin contact between an infant and parent. Since then lot of research work on kangaroo care has been done globally.

Manuscript received: $24^{\text {th }}$ Oct 2013

Reviewed: $29^{\text {th }}$ Oct 2013

Author Corrected: $15^{\text {th }}$ Nov 2013

Accepted for Publication: $29^{\text {th }}$ Nov 2013
These studies clearly depict advantages of kangarooing like weight gain in $\mathrm{KMC}$ babies, increased breast milk secretion and early discharge from NICU [5].

However, the effect of kangaroo care on physiological parameters is still controversial. Scanty research article is available on the effect of KMC on physiological variables [6-11]. Kangarooing promotes closeness of mother and baby making the baby to relax thus stabilizing heart rate and respiration rate of the baby. Based on this hypothesis we planned this observational study in a teaching hospital of central India. Thus the aim of our study was to determine the effect of kangaroo mother care on heart rate, respiratory rate and temperature in low birth weight babies

\section{Materials and Methods}

This hospital based observational study was conducted over the period of one year. Preterm babies weighing $<2000$ grams who got admitted in the NICU of pediatric department were included in the study. Included preterm babies were not having any neurological, cardiac and /or respiratory deficiencies. Babies with major congenital 
anomalies were also excluded from the study. There were 514 infants admitted during the data collection period. Of those 251 meeting the selection criteria, 138 were approached, 89 accepted to participate giving a refusal rate of 36 percent. So total 89 preterm babies were enrolled for the study. Out of these 89 babies, 9 babies were further excluded from the study because their mother refused to continue participating in the study after one day of kangarooing. Remaining 80 babies were enrolled for the study.

Kangarooing was provided to these babies from day one of delivery /admission to NICU or from the time they stabilized. KMC was done by the mother either in NICU or the adjacent room provided to mothers.

Mothers were doing kangarooing by either sitting on reclining chair or lying down on the hospital bed with the head rest raised to $45^{\circ}$.

Assessments were made before and after 30 minutes of kangaroo mother care daily for 3 consecutive days. Pre $\mathrm{KMC}$ heart rate (HR), respiratory rate (RR) and temperature was recorded just before starting KMC. Pre KMC measurement was done while newborn was on warmer or lying down on a cradle. Post KMC assessments were made after 30 minutes of kangarooing. The evaluations (respiratory rate, heart rate and temperature measurements) were done once a day, for three consecutive days and the mean values were taken for analysis.

For these evaluations a pulse oximeter (Stellar pulse rate monitor, manufactured by L\& T limited Mysore, India) and a mercury thermometer were utilized. The first assessment was done for breathing movements by counting the respiratory rate over one minute.

Next measurement was done for heart rate. For that a sensor was placed on the sole of the newborn's right toe and then heart rate was noted down. In the last the skin temperature was measured by keeping thermometer beneath the babie's axillary folds for three minutes.

The babies were fed one hour prior to starting Kangaroo Care. Most of the babies slept during Kangaroo Care and appeared relaxed.

Participation was voluntary and the mothers were allowed to withdraw from the study at any time. Informed consent was taken from all the mothers participating in the study.

\section{Results}

Out of 80 babies 46 babies were male and only $11 \%$ of the newborn were extremely LBW.

Table 1: Distribution of the newborn included in the study

\begin{tabular}{|c|c|c|c|c|c|c|c|c|}
\hline \multicolumn{2}{|c|}{ Weight at birth (grams) } & \multicolumn{5}{|c|}{ Gestational age (weeks) } & \\
\hline$<1200$ & $1201-1800$ & $1801-2000$ & $27-28$ & $29-30$ & $31-33$ & $34-36$ & AGA $^{*}$ & SGA $^{*}$ \\
\hline 6 & 39 & 35 & 5 & 15 & 21 & 39 & 63 & 17 \\
\hline $11 \%$ & $45 \%$ & $43 \%$ & $6 \%$ & $18 \%$ & $26 \%$ & $48 \%$ & $78 \%$ & $22 \%$ \\
\hline
\end{tabular}

*AGA-appropriate for gestational age

*SGA small for gestational age

Majority of the babies were above 1200 grams and two third of the babies were above 30 weeks (Table-1). Substantial numbers ( $78 \%$ ) of the babies were appropriate for gestational age while only $22 \%$ of babies were small for gestational age.

Table 2: Various parameters of the newborn included in the study

\begin{tabular}{|l|l|l|l|}
\hline $\begin{array}{l}\text { Mean Birth weight } \\
\text { (gm) } \pm \text { SD }\end{array}$ & $\begin{array}{l}\text { Mean gestational age } \\
\text { (weeks) }\end{array}$ & Mean age\# & Male: female ratio \\
\hline $1631.28 \pm 290.86$ & 32.18 & 9.15 days of life & $1.3: 1 \quad(46 / 34)$ \\
\hline
\end{tabular}

\# Age when kangaroo started

Mean gestational age of babies was 32.18 weeks and mean birth weight was $1631.28 \pm 290.86$ grams (Table-2). Mean postnatal age was 9.15 days of life when KMC was initiated. Male 46(57.5\%) babies outnumbered female babies. 
Table 3: Vital signs measured before and after 30 minutes of kangarooing

\begin{tabular}{|c|c|c|c|c|}
\hline Vital signs & Mean & SD & t-value & p-value \\
\hline \multirow{2}{*}{$\begin{array}{r}\mathrm{HR} / \text { minute Before KMC } \\
\text { After KMC }\end{array}$} & 140.48 & 12.46 & \multirow[t]{2}{*}{0.0968} & \multirow[t]{2}{*}{0.9230} \\
\hline & 140.66 & 12.04 & & \\
\hline \multirow{2}{*}{$\begin{array}{r}\mathrm{RR} / \text { minute Before } \mathrm{KMC} \\
\text { After KMC }\end{array}$} & 41.44 & 8.53 & \multirow[t]{2}{*}{0.4423} & \multirow[t]{2}{*}{0.6589} \\
\hline & 40.86 & 7.9 & & \\
\hline \multirow{2}{*}{$\begin{array}{l}\text { Before KMC } \\
\text { After KMC }\end{array}$} & 34.69 & 1.45 & \multirow[t]{2}{*}{13.983} & \multirow[t]{2}{*}{$<0.0001$} \\
\hline & 36.97 & 0.412 & & \\
\hline
\end{tabular}

HR-Heart Rate, RR- Respiratory Rate, Temp -Temperature measured over 3 minutes

Mean heart rate before starting KMC was140.48/minute and after 30 minutes of kangarooing HR was140.66/minute. Mean RR before starting KMC was 41.44/minute and after accomplishment of KMC means RR was 40/minute. These findings were statistically insignificant (Table-3).

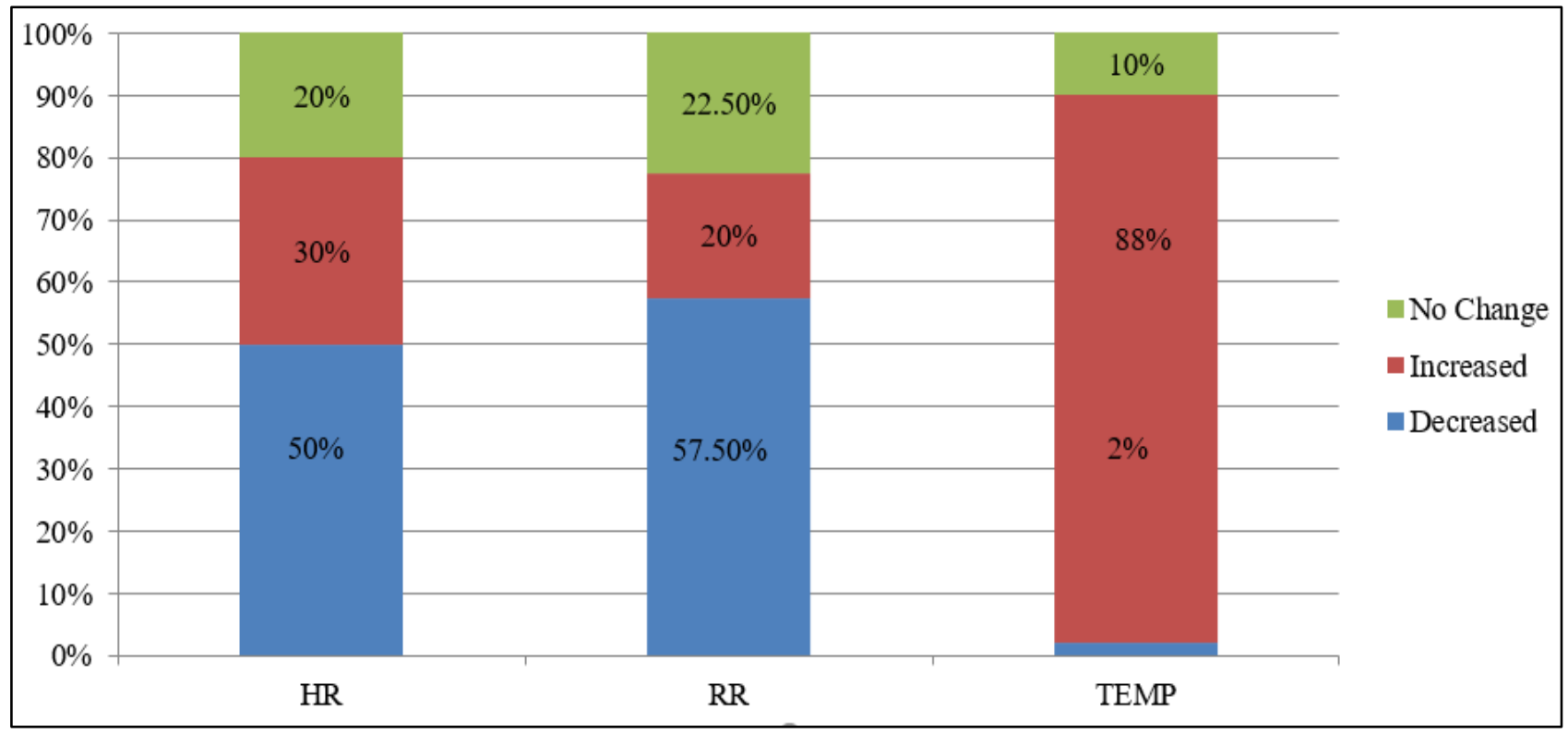

Figure 1: Showing percentages of babies with variation in vital signs

Table 4: Changes in heart rate Pre and Post KMC

\begin{tabular}{cccllll}
\hline Group & Newborn & Range & Mean \pm SD & Decreased & Increased & No change \\
\hline I & 6 & -7 to +5 & $1.5 \pm 5.6$ & $3(50 \%)$ & $2(33 \%)$ & $1(17 \%)$ \\
II & 39 & -8 to +5 & $-0.4 \pm 3.4$ & $20(51.28 \%)$ & $7(18 \%)$ & $12(30.7 \%)$ \\
III & 35 & -6 to +5 & $-0.17 \pm 3.5$ & $17(49 \%)$ & $15(43 \%)$ & $3(8 \%)$ \\
\hline Total & 80 & -8 to +5 & & $40(50 \%)$ & $24(30 \%)$ & $16(20 \%)$ \\
\hline
\end{tabular}

In $50 \%$ of babies heart rate was decreased after kangarooing while HR was increased in $30 \%$ of babies. No change was noticed in the HR of $20 \%$ of babies after half an hour of kangarooing (Table-4). Maximum gain in heart rate was 5 beat/minute whereas maximum fall in heart rate was 8 beats $\backslash$ minute. Changes in heart rate were within normal limit and they were statistically insignificant. 
Table 5: Changes in respiratory rate Pre and Post KMC

\begin{tabular}{|c|c|c|c|c|c|c|c|}
\hline Group & $\begin{array}{l}\text { No. } \\
\text { infants }\end{array}$ & of & Range & Mean \pm SD & Decreased & Increased & No change \\
\hline I & 6 & & -6 to +1 & $-3.5 \pm 3.15$ & $4(66.67 \%)$ & $1(16.67 \%)$ & $1(16.67 \%)$ \\
\hline II & 39 & & -3 to +2 & $-2 \pm 2.16$ & $22(56.41 \%)$ & $615.38 \%)$ & $11(28.2 \%)$ \\
\hline III & 35 & & -5 to +5 & $-0.6 \pm 2.9$ & $20(5.14 \%)$ & $9(26 \%)$ & $6(17 \%)$ \\
\hline Total & 80 & & -6 to +5 & & $46(57.5 \%)$ & $16(20 \%)$ & $18(22.5 \%)$ \\
\hline
\end{tabular}

Table -5 Shows that $57.5 \%$ of babies showed decreased RR. Alterations in respiratory rate were within normal limit. No change in RR was seen in $22.5 \%$ of babies after half an hour of kangaroo care. All the changes were statistically insignificant. Maximum increase in $\mathrm{RR}$ was 5/minutes whereas maximum decrease in RR was 6/minute.

Table 6: Change in skin temperature Pre and Post KMC

\begin{tabular}{lcc}
\hline & Before KMC No. of neonates & After KMC No. of neonates \\
\hline Normothermic * & $12(15 \%)$ & $77(96.2 \%)$ \\
Hypothermic \# & $66(82.5 \%)$ & $2(2.5 \%)$ \\
Hyperthermic \$ & $2(2.5 \%)$ & $1(1.2 \%)$ \\
\hline
\end{tabular}

Temperature was measured by keeping mercury thermometer over axilla of newborn for 3 minutes.

*skin temperature $36.5--37.5^{\circ} \mathrm{C}$

\#skin temperature $<36.4 .0^{\circ} \mathrm{C}$

\$skin temperature $>37.6{ }^{\circ} \mathrm{C}$

It is evident from Table-6 that before $\mathrm{KMC} 82.5 \%$ of babies were hypothermic but after $\mathrm{KMC} 96.2 \%$ babies became normothermic within half an hour which was found highly significant ( $p$-value $<0.0001$ ).

\section{Discussion}

Our results show that heart rate either increased by 5 beats/ minute or decreased by 8 beats/ minute within normal limit; however, this finding is not statistically significant. In the current study decrease in mean HR were of greater magnitude than increase in HR.

This can be explained by the fact that babies, both preterm and full-term, cry less when placed in skin to skin contact with their mothers. Kangarooing also increases sleep time, as well as time spent in quiet sleep [12].

Decreased crying means less stress and subsequently lower levels of cortisol. Randomized controlled trial done by mooncey et al on stable premature infants have shown reductions in cortisol levels by $60 \%$ or more under the influence of 20 minutes or more of Kangaroo care when compared with infant left in an incubator [13].
During KMC the c-afferent nerves of the mother's and infant's chest surfaces respond to the pleasing human touch of KMC and send the pleasing touch message straight to the insular cortex of the limbic area of the brain. Upon pleasing touch stimulation, oxytocin is released within the brain and travels along neurons to14 different areas of the brain.

The first target is the brain stem where oxytocin immediately calms and stabilizes cardio-respiratory variables as the brain stem shifts from sympathetic (stress, hyper alertness and reactivity, sense of threat) to parasympathetic (relaxation, calm, contentment, safety) control. The increase in the heart rate may occur because of the change in the newborn's body position from supine to vertical and the handling, which leads to increased stress for the newborn. Heart rate may rise by $5-10$ beats per minute during kangarooing rising initially in response to the head 
Research Article

tilting upward and later due to infant warming [14,15]. Some researchers reported no or minimal change in heart rate during $\mathrm{KMC}$. Whereas others reported increased heart rates within normal limit [16]. The findings of the study revealed that respiratory rate either increased by $5 /$ minute or decreased by $6 /$ minute during $\mathrm{KMC}$ which was within normal limit having no statistical significance. A possible explanation for the decreased respiratory rate is based on the upright position of the infant.

This method allows the infant to be held in a ventral position at an angle of $\sim 60$ degrees which decreases the compression of the diaphragm. Ventilation and perfusion are gravity dependent, so an upright position optimizes respiratory function. However, the heart and breathing rates are uncertain data, because the mother's respiratory and cardiac patterns may be superimposed on the newborn

Research done by Fohe et al showed that respiratory rate was significantly decreased during kangaroo care whereas results of Tonhage $\mathrm{CB}$ and Fischer $\mathrm{CJ}$ are suggestive of minimal change during kangaroo mother care [17-19].

The results of our study regarding temperature control during $\mathrm{KMC}$ are consistent with various previous research and showing that $\mathrm{KMC}$ is highly favorable to newborn in controlling body temperature $(\mathrm{p}<0.0001)[1,14,17]$. A mother has actual "thermal synchrony" with her infant. When a baby is placed in the kangaroo position, skin to skin with his mother, the temperature of the mother's breasts actually change so that her baby can better maintain his own temperature. If the baby gets too cold the mother's body temperature will actually warm up one degree to aid warm the baby [14]. If the baby gets too hot, the mother's body temperature will decrease one degree to cool the baby. This even works when twins are kangarooed [15].

This has been proved by various studies that in an upright prone position babies tolerate noise and activity around them much better [20].

Furthermore, the flexed position that the baby assumes on his mother's chest as opposed to lying on his back in an incubator is a more efficient position for conserving heat.

The extra sleep that the infant gets snuggling with mom and the assistance in regulating body temperature helps the baby conserve energy and redirects calories expenditures toward growth and weight gain. In short it can be stated that Kangarooing provides relief from tense NICU milieu, enhances bonding and eases physiologic transitions.

\section{Conclusion}

This study concludes that Kangaroo method produces little variation in heart rate and respiratory rate that also within normal limit with optimum temperature control. Further

randomized controlled trials are required to find out the effect of kangaroo mother on heart rate and respiratory rate.

\section{Funding: Nil \\ Conflict of interest: Nil \\ Permission from IRB: Yes}

\section{References}

1. J. E. Lawn, S. Cousens, and J. Zupan, “4 Million neonatal deaths: when? Where? Why?" The Lancet, vol. 365, no. 9462, pp. 891-900, 2005.

2. J. E. Lawn, S. Cousens, Z. A. Bhutta et al., "Why are 4 million newborn babies dying each year?"The Lancet, vol. 364, no. 9432, pp. 399-401, 2004.

3. National Health Profile Report, Central Bureau of Health Intelligence, Ministry of Health and Family Welfare, pp. 916,2010, http://cbhidghs.nic.in/writereaddata/mainlinkfile/fi le1131.pdf.

4. Charpak N, Ruiz-Pelaez JG, Charpak Y. Rey-Martinez Kangaroo Mother Program: an alternative way of caring for low birth weight infants? One year mortality in a two cohort study. Pediatrics. 1994;94(6 Pt 1):804-10.

5. Ramanathan K, Paul VK, Deorari AK, Taneja U, George Kangaroo Mother Care in very low birth weight infants. Indian J Pediatr 2001; 68: 1019-23.

6. Neu M, Browne JV, Vojir C. The impact of two transfer techniques used during skin-to-skin care on the physiologic and behavioral responses of preterm infants. Nurs res 2000 Jul-Aug;49 (4):215-23.

7. Bosque EM, Brady JP, Affonso DD, Wahlberg V Physiologic measures of kangaroo versus incubator care in a tertiary-level nursery. J Obstet Gynecol Neonatal Nurs. 1995 Mar-Apr; 24(3):219-26.

8. Bohnhorst B, Gill D, Dördelmann M, Peter CS, Poets $\mathrm{CF}$. Bradycardia and desaturation during skin-to-skin care: no relationship to hyperthermia. J Pediatr. 2004 Oct;145(4):499-502.

9. Ludington-Hoe SM, Thompson C, Swinth J, Hadeed AJ, Anderson GC. Kangaroo care: research results, and practice implications and guidelines. Neonatal Netw. 1994 Feb;13(1):19-27.

10. Bulfone G, Nazzi E, Tenore A. [Kangaroo Mother Care and conventional care: a review of literature].[Article in Italian] .Prof In ferm 2011 Apr-Jun; 64(2):75-82.

11. Boju SL, Gopi Krishna M, Uppala R, Chodavarapu $\mathrm{P}$, Chodavarapu R. Short spell kangaroo mother care and its differential physiological influence in subgroups of preterm babies. Journal of Tropical Pediatric 2012 Jun; 58(3):189-93.doi:10.1093/tropej/fmr072.Epub 2011 Sep 9 
12. Ludington-Hoe SM, Johnson MW, Morgan K, Lewis T, Gutman J, Wilson PD, Scheir MS. Neurophysiological assessment of neonatal sleep organization: Preliminary results of a randomized, controlled trial of skin contact with preterm infants. Pediatrics 2006;117(5):e909-23.

13. Mooncey S, Giannakoulopoulos X, Glover V, Acolet D, Modi N. The effect of mother -infant skin-to-skin contact on plasma cortisol and beta-endorphin concentrations in preterm infants. Infant Behav Dev. 1997;20(4):553-557.

14. Schrod L, Walter J. Effect of head-up tilt position on autonomic function and cerebral oxygenation in preterm infants. Biol Neonate. 2002; 81: 255-259.

15. Ludington-Hoe SM, Nguyen N, Swinth J, Satyshur R. Kangaroo care compared to incubators in maintaining body warmth in preterm infants. Biol Res Nurs. 2000;2(1):60-73.

16. Fischer, C.Sontheimer, D.,Scheffer, F., Bauer, J., \& Linderkamp, O. (1998). Cardiorespiratory stability of premature boys and girls during kangaroo care. Early Human Development, 52(2), 145-153.

17. Föhe, K., Kropf, S, \& Avenarius, S. (2000). Skin-to-skin contact improves gas exchange in premature infants. Journal of Perinatology, 20(5), 311-315.

18. Tornhage CJ, Stuge E, Lindberg T, Serenius F. First week kangaroo care in sick very preterm infants. Acta Paediatr. 1999;88(12):1402-1404.

19. Ludington-Hoe SM, Anderson GC, Swinth JY, Thompson C, Hadeed AJ (2004). Randomized controlled trial of kangaroo care: Cardiorespiratory and thermal effects on healthy preterm infants. Neonatal Netw. 23:3948.

20. Ludington-Hoe, S. Kangaroo Care: The Best You Can Do to Help Your Preterm Infant. New York: Bantam Books, 1993.

\section{How to cite this article?}

Verma P, Verma V. Effect of Kangaroo Mother Care on Heart rate, Respiratory rate and Temperature in Low Birth Weight Babies. Int J Med Res Rev 2014;2(2):80-85. doi:10.17511/ijmrr.2014.i02.02 